\title{
Auf den Rahmen kommt es an!
}

\begin{abstract}
Ende der achtziger Jahre hat sich angesichts der wachsenden Umweltprobleme die Forderung nach einer umweltorientierten Produktgestaltung durchgesetzt. Unternehmen haben eine Verantworfung für den gesamten Lebensweg der Produkte; diese sollen möglichst im Kreislauf geführt werden. Sind Konzepte zur Nutzungsintensivierung und Lebensdaververlängerung eine geeignete Strategie? Anhand zweier Fallbeispiele werden Potentiale und Grenzen aufgezeigt.
\end{abstract}

$\mathrm{D}$

Von Beate Stabl und Jïrgen Fleig as Kreislaufwirtschafts- und Abfallgesetz faßt einige Konzepte zur Nutzungsintensivierung und Lebensdauerverlängerung von Produkten unter dem Begriff Produktrecycling zusammen. Idealtypisch lassen sich drei Konzepte unterscheiden:

1. Zur Nutzungsintensivierung werden die Produkte nicht mehr an einen Kunden verkauft, sondern vermietet bzw. von mehreren Kunden genutzt; damit sind Konzepte der ökoeffizienten Dienstleistungen gemeint (1).

2. Die Lebensdauer der Produkte kann durch Wartung und Instandsetzung oder durch Beratung zum richtigen Gebrauch verlängert werden.

3. Wenn der Erstnutzer das Produkt nicht mehr haben will, kann es - auf einer hohen Wertschöpfungsstufe - aufgearbeitet, modernisiert und anschließend wieder verkauft werden.

Diese hochwertigen Formen der Kreislaufwirtschaft können dazu beitragen, Energie- und Ressourcenverbräuche und damit auch die Emissionen und Abfälle in die Umwelt zu reduzieren. Zugleich scheinen sie für Unternehmen auch ökonomisch attraktiv zu sein. Bei geeigneten Rahmenbedingungen von Seiten des Marktes eröffnen sich dadurch Geschättsfelder für „neue" Dienstleistungen in der Kreislaufwirtschaft. Im Rahmen des Modellprojektes PROKREIS haben sich deshalb vier Unternehmen zum Ziel gesetzt, Konzepte zur Nutzungsintensivierung und Lebensdauerverlängerung ihrer Produkte zu entwickeln und umzusetzen bzw. ihre bestehenden Lösungen auszubauen und ökonomisch und ökologisch effizienter zu machen (2). In zwei Fällen unterzog das Fraunhofer-Institut für Systemtechnik und Innovationsforschung diese Konzepte einer ökologischen Prüfung. Die Analysen sollten weniger die letzte Stelle hinter dem Komma der jeweiligen
Umweltwirkungen ermitteln, als vielmehr aufzeigen, wo das wesentliche Gestaltungspotential zur Entlastung der Umwelt liegt und entsprechende Maßnahmen in den Firmen motivieren.

Für die Fragestellung, ob Konzepte zur Nutzungsintensivierung und Lebensdauerverlängerung auch ökologische Entlastungseffekte erzielen, ist eine umfassende Betrachtung über den ganzen Lebensweg erforderlich. Dies soll verhindern, daß es zu Verlagerungen von Umweltproblemen von einem Umweltmedium in ein anderes oder beispielsweise von der Herstellung zur Nutzung bzw. Entsorgung kommt. Deshalb sollen auch bei der Analyse nicht nur die Inputgrößen (Ressourcen, Energie) sondern auch die Outputseite (Emissionen in Wasser, Luft und Boden, Abfälle) betrachtet werden. Methoden für solch eine Betrachtung sind Ökobilanzen, der MIPS-Ansatz und der kumulierte Energieverbrauch (KEA). Während Ökobilanzen eine Beurteilung sämtlicher Input- und Outputgrößen zum Ziel haben (3), beschränken sich andere Methoden auf einzelne Kenngrößen. Für das MIPS-Konzept ist der Ressourcenverbrauch entscheidend (4), für den KEA-Ansatz der kumulierte Energieverbrauch (5). Je nach Fragestellung und produktspezifischen Voraussetzungen haben die Methoden ihre Vor- und Nachteile.

\section{Von der Wiege bis zur Bahre}

Die Produkte der Firma EconAir AG, Lübbecke, sind technische Sprays in Aerosoldosen im Mehrwegsystem. Die Produktpalette reicht von Reinigungs- und Pflegemitteln über Trenn- und Gleitmittel bis hin zu Elektronik- und Elektroreinigern. Mittels einer Spezialtechnik werden die Dosen kontrolliert entleert, getestet und wiederbefïllt. Durch die Wiederverwendung der Dosen sind zusätzliche Transportaufwendungen (Rücktransport) notwendig. Diese müssen der Neuproduktion der Weißblechdosen bei dem Ein- wegsystem gegenübergestellt werden. Zwar würde für den Transport eine Kenngröße Energieverbrauch genuigen, aber bei dem Herstellungsprozeß der Weißblechdosen ist eine zusätzliche Abschätzung der Outputgrößen sinnvoll. Daher bietet sich eine ökobilanzielle Betrachtung für den Vergleich der Verpackungssysteme an.

Fazit der durchgeführten Beurteilung ist, daß die Vorteilhaftigkeit des Mehrwegsystems stark von der Rücklaufquote abhängt. Bei dem Beispiel ist das Mehrwegsystem ab einer Rücklaufquote von ca. 15 Prozent in fast allen Parametern besser als die Einwegvariante. Eine Ausnahme sind die VOC-Emissionen, die nur vom Transport herrühren. Dieser Vorteil der Mehrwegvariante gegenüber der Einwegvariante wird mit steigender Rücklaufquote immer deutlicher. Daher müssen Maßnahmen ergriffen werden, um die Rücklaufquote, d. h. das Produktrecycling zu erhöhen. Untersuchungen von EconAir zeigen, daß je nach Produktgruppe bzw. Abnehmer unterschiedliche Rücklaufquoten erreicht werden. Maßnahmen zur Erhöhung der Akzeptanz des Mehrwegsystems bei den Kunden wären sinnvoll. In PROKREIS wurden deshalb eingehende Markt- und Vertriebsanalysen durchgeführt, um sowohl den Verkäufern als auch den Kunden die Wichtigkeit der Riickgabe zu vermitteln. Es soll nun ein produktbegleitendes Informationssystem entwickelt werden, mit dessen Hilfe Kundengruppen identifiziert werden können, die eine geringe Rücklaufquote haben; sie sollen dann durch gezielte Aktionen zur Steigerung des Rücklaufs bewegt werden.

Mit der Zunahme der Rücklaufquote wird der Transport immer wichtiger. Eine Optimierung der Logistik (Fahrzeugauswahl, Auslastung, Strecke) ist daher anzustreben. Beispielsweise soll in Zukunft durch den Einsatz eines neuen Kastens das Gewicht der Transportverpackung der Dosen gesenkt werden. Schwierig zu quantifizieren, aber sicher von einiger Bedeutung ist die kontrollierte Entsorgung der Dosen und vor allem der Inhaltsstoffe bei dem Mehrwegsystem; Einwegdosen werden nicht selten noch mit einem mehr oder weniger beträchtlichen Anteil an Inhaltsstoffen ,weggeschmissen“.

\section{Mieten staft kaufen}

Die Mercedes-Benz CharterWiay GmbH, Berlin, vermietet Nutzfahrzeuge an Speditionen, Paketund Zustelldienste, Rettungsdienste und als Müllfahrzeuge an öffentliche oder private Entsorger. CharterWay organisiert für den Kunden 
die ganze Wartung und Instandhaltung und besorgt bei Bedarf auch Ersatzfahrzeuge (Flottenmanagement). Es wird also eine „Verkauf von Nutzen“- statt einer ,Verkauf von Produkten"-Strategie verfolgt. CharterWay behält die Eigentumsrechte und vermietet die Fahrzeuge für drei bis vier Jahre an den Kunden. Da Untersuchungen für den Fahrzeugbereich gezeigt haben, daß die Nutzungsphase und damit der Kraftstoffverbrauch entscheidend ist, wurde eine ökologische Bewertung an Hand des kumulierten Energieverbrauchs durchgeführt (6).

Die Vorteile der Variante „Mieten“ bestehen vor allem darin, daß erstens der Kunde weniger Ersatzfahrzeuge bereit halten muß und zweitens bei einer intensiven Nutzung, $d . h$. hoher Fahrleistung, die Fahrzeuge früher durch effizientere Fahrzeuge ersetzt werden und dadurch der Energieverbrauch gesenkt werden kann.

Diese Einsparung in der Nutzungsphase kompensiert jedoch nicht den Herstellungsaufwand für das neue effizientere Fahrzeug, so daß es notwendig ist, die Fahrzeuge noch in einer zweiten Vermietung - jedoch mit geringerer Fahrleistung weiter zu gebrauchen. Der Vorteil des Mietkonzeptes nimmt $a b$, wenn der Unterschied in der Fahrleistung zwischen erster und zweiter Nutzungsphase kleiner wird. Er steigt, wenn die Technologiesprünge zur Senkung des Kraftstoffverbrauchs größer werden.

Deutlich wurde, daß durch den Einsatz effizienterer Technologie und eine „Kaskaden“-Nutzung, bei der die effizienteste Technologie bei hoher Fahrleistung eingesetzt und dann bei geringerer Fahrleistung weitergenutzt wird, energetische Einsparpotentiale möglich sind.
Solch ein Vorgehen wird durch eine „Verkauf von Nutzen"-Strategie erst möglich, da durch lange Amortisationszeiten bei einem Kauf die Fahrzeuge nicht so früh ersetzt werden können. Gleichzeitig sind aber auch die Grenzen sichtbar. Sobald nur noch geringe Verbesserungen der Effizienz möglich sind, werden die Einsparpotentiale aus der Nutzungsphase minimal.

Aufgrund dieser Ergebnisse plant CharterWay nun die Einführung einer zweiten Mietphase. Wurden die Fahrzeuge bislang für vier Jahre vermietet und anschließend als Gebrauchtfahrzeuge verkauft, soll es nun zwei Vermietungen von jeweils drei Jahren geben. In der ersten Phase sollen die Fahrzeuge möglichst intensiv, d. h. mit hoher Jahreskilometerleistung genutzt werden. In der zweiten Phase sollen die Fahrzeuge eine geringere jährliche Laufleistung haben.

\section{- Fazif: weiterer Klärungsbedarf}

Wurden bisher den Konzepten zur Nutzungsintensivierung und Lebensdauerverlängerung $z$. T. per se ökologische Entlastungseffekte zugeschrieben, zeigen die Beispiele, daß nur unter bestimmten Rahmenbedingungen die Konzepte ökologische Entlastungen mit sich bringen. Um diese Einflußfaktoren einer erfolgreichen Strategie zur Reduktion von Stoffströmen und Umweltbelastungen $\mathrm{zu}$ identifizieren, müssen aber noch mehr Praxisbeispiele im einzelnen bewertet werden. Ziel sollte eine Klassifizierung der Strategien mit ihren Möglichkeiten und Grenzen sein. Auch muß diskutiert werden, welche Folgen ein in Zukunft immer geringer werdender Effizienzgewinn auf solche Strategien hat.

\section{Anmerkungen}

(1) Hockerts, Kai, Arnd Permecky, Stefan Hauch, Stefan Seuring, Stefan Schweitzer (Hrsg.): Kreislaufwirtschaft statt Abfallwirtschaft. Optimierte Nutzung und Einsparung von Ressourcen durch Öko-Leasing und Servicekonzepte. Schriften der Bayreuther Initiative für Wirtschaftsökologie e.V., Ulm 1995.

(2) Das Verbundprojekt PROKREIS, „Industrielle Konzepte zur Nutzungsintensivierung und Lebensdauerverlängerung von Produkten", wurde vom Bundesforschungsministerium im Rahmen des Programms Produktion 2000 gefördert. Vgl. zu den Ergebnissen auch Fleig, Jürgen: Höherer Nutzen und längeres Leben: Neue Produktkonzepte für eine kreislauforientierte Wirtschaft. In: io Management, 66. Jg. (1997), Heft 4, S. 38-43.

(3) ISO 14040: Umweltmanagement - Ökobilanz - Prinzipien und allgemeine Anforderungen. 1997.

(4) Wuppertal Institut für Klima, Umwelt, Energie: Die MIPS Ergebnisse - Ökologische Rucksäcke. Online in Internet: URL: http://www.wupperinst.org/Projekte/ mipsonline/index.html. 16.03.99.

(5) Verein Deutscher Ingenieure: VDI-Richtlinie 4600. Kumulierter Energieaufwand. Begriffe, Definitionen, Berechnungsmethoden. Düsseldorf 1997.

(6) Hoffmann, C.: Kumulierter Energieaufwand und energieoptimierte Nutzungsdauer von Personenkraftwagen. München 1995.

\section{Die Autorlnnen}

Dr. Beaie Stahl und Dr. Jürgen Fleig sind wissenschaftliche Mitarbeiter am Fraunhofer-Institut für Systemtechnik und Innovationsforschung (ISI). Kontakt: ISI, Breslaver Str. 48, 76139 Karlsruhe. Tel. 0721/6809-306, Fax -131,

E-mail: jf@isi.fhg.de

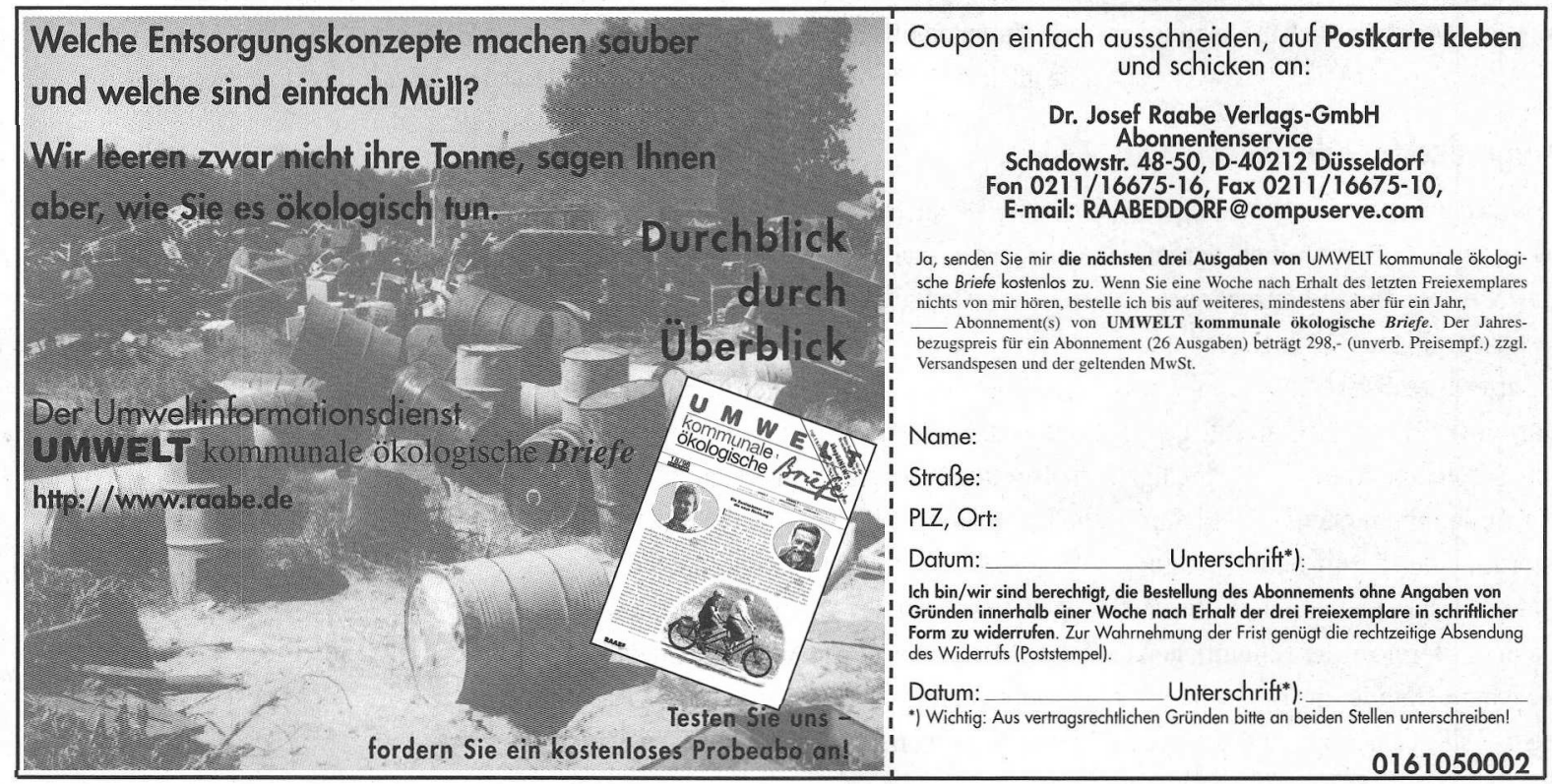


(c) 20I0 Authors; licensee IÖW and oekom verlag. This is an article distributed under the terms of the Creative Commons Attribution Non-Commercial No Derivates License (http://creativecommons.org/licenses/by-nc-nd/3.o/), which permits unrestricted use, distribution, and reproduction in any medium, provided the original work is properly cited. 